\title{
MODELAGEM DA DISTRIBUIÇÃO DIAMÉTRICA EM POVOAMENTOS DE EUCALIPTO SUBMETIDOS A DESBASTE UTILIZANDO AUTÔMATOS CELULARES ${ }^{1}$
}

Daniel Henrique Breda Binoti², Mayra Luiza Marques da Silva Binoti ${ }^{3}$, Hélio Garcia Leite ${ }^{4}$, Antonilmar Silva ${ }^{5}$ e Ana Carolina de Albuquerque Santos ${ }^{6}$

\begin{abstract}
RESUMO - Este estudo apresenta um modelo de distribuição diamétrica baseado em um modelo de Autômatos celulares (AC) unidimensionais e redes neurais artificiais (RNA) para a simulação de desbaste. Cada célula do AC proposto representa uma classe de dap, sendo o estado futuro previsto em função do estado atual dessa célula, do estado de suas quatro células vizinhas e de sua idade atual e futura. Como regra de evolução, utilizou-se uma RNA. A exatidão foi avaliada empregando-se o procedimento estatístico L\&O relação entre frequências observada e estimada e realismo biológico do modelo construído. Entre as redes treinadas, foram selecionadas as 10 que representavam a evolução da distribuição diamétrica com maior exatidão. Entre essas 10 RNA, sete apresentaram valores estimados estatisticamente iguais aos observados ( $\mathrm{p}>0,01)$. O enfoque de modelagem proposto permite estimar distribuições diamétricas futuras com exatidão.
\end{abstract}

Palavras-chave: Inteligência computacional, Redes Neurais Artificiais e L\&O.

\section{MODELING THE DIAMETER DISTRIBUTION OF THINNED EUCALYPTUS STANDS USING A CELLULAR AUTOMATA}

\begin{abstract}
This study presents a diametric distribution model based on a one-dimensional cellular automata (CA) model and artificial neural network (ANN). Each CA cell proposed stands for a dap class, with the future state being predicted in function of the present state of this cell, the state of its surrounding cells and of its present and future ages. An ANN was used as a rule of evolution. Accuracy was evaluated by applying the statistical procedure $L \& O$, a relationship between observed and estimated frequency and biological realism of the model built. Of the trained networks, 10 representing diametric distribution evolution with more accuracy were selected. Of these 10 ANR, seven represented estimated values statistically equal to those observed ( $p>0.01)$. The focus of the modeling proposed allows estimating future diametric distributions accurately.
\end{abstract}

Keywords: Computational intelligence, Artificial neural network and $L \& O$.

\section{INTRODUÇÃO}

A estimação da distribuição diamétrica de povoamentos equiâneos é essencial para a avaliação econômica de multiprodutos da madeira. Essa estimação é comumente realizada por modelos de distribuição diamétrica. O procedimento utilizado nesses modelos se baseia no ajuste de uma função densidade probabilidade $(f d p)$, aos dados de parcelas permanentes, e no emprego de modelos de regressão relacionando os parâmetros dessa $f d p$ com características do povoamento (CLUTTER et al., 1983; CAMPOS; LEITE, 2009).

Diferentes funções (NELSON, 1964; BLISS; REINKER, 1965; CLUTTER; BENNETT, 1965; HAFLEY;

\footnotetext{
${ }^{1}$ Recebido em 01.10.2010 aceito para publicação em 04.06.2012.

${ }^{2}$ Doutorado na Universidade Federal de Viçosa, Departamento de Engenharia floresta. E-mail: <danielhbbinoti@ gmail.com>.

${ }^{3}$ Universidade Federal dos Vales do Jequitinhonha e Mucuri, Departamento de Engenharia Florestal. E-mail: <MayraBinoti@gmail.com>.

${ }^{4}$ Universidade Federal de Viçosa, Centro de Ciências Agrárias, Departamento de Engenharia Florestal. E-mail: <hgleite@ gmail.com>.

${ }^{5}$ Celulose Nipo-Brasileira. E-mail: <antonilmar.silva@ cenibra.com.br>.

${ }^{6}$ Programa de Pós-Graduação em Ciencia Florestal pela Universidade Federal de Viçosa, Departamento de Engenharia Florestal, UFV.E-mail: <anaflorestaufv@gmail.com>.
} 
SCHUREUDER, 1977; PALAHÍ et al., 2007; BAILEY; DELL, 1973; NOGUEIRA et al., 2005) e formas de obtenção dos seus parâmetros em idades futuras são utilizadas para a caracterização da distribuição diamétrica de povoamentos equiâneos (CAO, 2004). A função hiperbólica destaca-se entre as $f d p$ em razão, principalmente, da sua flexibilidade, facilidade de ajuste e correlação com parâmetros do povoamento (GUIMARÃES, 2002; CAMPOS; LEITE, 2009; LEITE et al., 2010).

Técnicas de inteligência computacional, como redes neurais artificiais (DIAMONTOPOULOU, 2005; GÖRGENS, 2006; SILVA, 2008), e de vida artificial, como autômatos celulares (MARTHEY et al., 2007), tem sido utilizadas em algumas aplicações na área florestal. Redes neurais artificiais (RNA) são processadores paralelamente distribuídos compostos por unidades de processamento simples (neurônios artificiais), que armazenam conhecimento experimental (aprendizagem), tornando-o disponível para uso (generalização) (BRAGA et al., 2000; HAYKIN, 2001). Autômatos celulares $(A C)$ são sistemas de interações locais, discretos no tempo e no espaço, compostos de células, onde o estado futuro de cada célula é resultado de seu estado atual, da sua regra de transição e da sua interação com células vizinhas (WOLFRAM, 1983; ILACHINSKI, 2001). As células são componentes simples que interagem localmente e a complexidade em âmbito global advém de regras entre essas unidades mais simples. Diversos métodos podem ser utilizados como regras de transição, com destaque para RNA (HAYKIN, 2001).

A modelagem empregando AC tem sido feita para a descrição de sistemas complexos, como fenômenos químicos (d’HUMIERES; LALLEMAND, 1986; GERHARDT; SCHUSTER, 1989), crescimento vegetal (LINDEMAYER; ROZENBERG, 1976), crescimento de cristais (KESSLER, 1990), ecologia (PHIPPS, 1992), propagação de doenças infecciosas (SEGEL, 1999), dinâmica social (AXTELL; EPSTEIN, 1996) e incêndios florestais (BAK et al., 1990).

O objetivo deste estudo foi de construir, testar e avaliar um modelo de autômatos celulares, com sua regra de evolução baseada em redes neurais artificiais, para a projeção da distribuição de diâmetros em povoamentos de eucalipto e a simulação de desbastes.

Revista Árvore, Viçosa-MG, v.36, n.5, p.931-939, 2012

\section{MATERIAIS E MÉTODOS}

\section{Dados}

Os dados utilizados neste estudo foram obtidos de 400 parcelas retangulares permanentes com área de $340 \mathrm{~m}^{2}$, instaladas em povoamentos de híbridos de Eucalyptus grandis x Eucalyptus urophylla, na região Centro Oeste do Estado de Minas Gerais, Brasil. Esses povoamentos foram estabelecidos sob arranjo espacial de 3 x 3 m, com ciclo de corte médio de 7 anos, sendo a madeira utilizada para produção de polpa de celulose de fibra curta, com produtividade média, na idade de corte, de $40 \mathrm{~m}^{3} \mathrm{ha}^{-1} \mathrm{ano}^{-1}$. As medições de diâmetros foram efetuadas em todas as árvores com dap acima de $5 \mathrm{~cm}$, nas idades médias de 28, 40, 52, 64, 76 e 88 meses. O arquivo de dados foi separado em dois subconjuntos, sendo um para construção do modelo ( $50 \%$ dos dados) e o outro para sua validação. Nessa separação foi mantida uma frequência homogênea de dados por classe de capacidade produtiva.

Para a simulação de desbastes, ajustou-se um modelo $\mathrm{dap}=\mathrm{e}^{\left(\beta_{0}+\frac{\beta_{1}}{\text { idade }}\right)}$, para cada árvore de cada parcela, em que $\beta(i=0,1)$ são os parâmetros do modelo. Com os parâmetros das equações ajustadas para cada árvore, foram simuladas aleatoriamente cinco intensidades de desbastes variando entre 20 e $50 \%$ da área basal em idades variando de 30 a 150 meses, sendo o corte final projetado para os 180 meses, para facilitar a simulação de desbastes.

\section{Modelo de Autômatos Celulares}

Para um modelo unidimensional de AC, o valor da primeira célula no tempo $t$, denominado $c_{i}(t)$, evolui de acordo com a regra $\mathrm{F}$, que é função do $c_{i}(t)$ e das outras células que se encontram dentro da extensão $r$ (para ambas as direções) de $c_{i}(t)$ (ILACHINSKI, 2001), isto é:

$$
\mathrm{C}_{\mathrm{i}}(\mathrm{t})=\mathrm{F}\left(\mathrm{C}_{\mathrm{i}-\mathrm{r}}(\mathrm{t}-1), \ldots, \mathrm{C}_{\mathrm{i}-\mathrm{r}}(\mathrm{T}+1)\right)
$$

$\mathrm{O}$ AC proposto neste estudo é unidimensional, e cada célula possui quatro vizinhos $(r=2)$. As células representam uma classe de diâmetro de $1 \mathrm{~cm}$ de amplitude, sendo o estado de cada célula corresponde à probabilidade de ocorrência de árvores nessa classe.

Para a construção da regra de evolução do AC foram utilizadas redes neurais artificiais, com as seguintes estruturas: (1) O arquivo de dados foi formatado com 
simulações aleatórias de desbastes. As redes foram treinadas, tendo como variáveis de entrada - estado atual da célula, estado das células contidas na extensão da vizinhança, intensidade de desbaste e as idades atual e futura. O desbaste foi utilizado como variável endógena do sistema. (2) variáveis de entrada estado atual da célula, estado das células contidas na extensão da vizinhança e as idades atual e futura. A projeção e treino das redes nesse caso foram realizados até a idade do desbaste e após o desbaste, com a mesma rede. (3) variáveis de entrada - estado atual da célula, estado das células contidas na extensão da vizinhança e as idades atual e futura. A projeção e treino das redes nesse caso foi realizada até a idade do desbaste (3a) e a após o desbaste (3b) com outra rede. A estrutura de formatação dos dados para treinamento das redes é apresentada na Tabela 1. Em todas as metodologias propostas, projetou-se o estado futuro da classe de dap (célula).

Foram testados diferentes arquiteturas e tipos de redes, sendo selecionada aquela que melhor representava os dados. Foram testadas redes de função de base radial (RBF Radial Basis Function), perceptrons de múltiplas camadas (MLP Multilayer perceptron) e perceptrons de camada única (Perceptron), com diferentes arquiteturas, e para cada metodologia foram treinadas 10 redes de cada tipo. O software Statistica 8 foi utilizado nessa etapa do estudo.

\section{Modelo de distribuição diamétrica}

Para avaliação das projeções realizadas pelo modelo de AC empregado neste estudo, realizou-se a projeção da distribuição diamétrica por um modelo de distribuição diamétrica comumente utilizado. Utilizou-se como função densidade a probabilidade da função hiperbólica (hyper) descrita por Guimarães (2002) e pode ser descrita como:

$$
f(X)=\left\{\omega \frac{\gamma}{\beta}\left(\frac{(X-\alpha)}{\beta}\right)^{(\gamma-1)}\left(1-\tanh \left(\left(\frac{(X-\alpha)}{\beta}\right)^{\gamma}\right)^{2}\right)\right.
$$

em que $\omega$ é o valor assintótico, $\alpha$ o parâmetro de locação, $\beta$ o parâmetro de escala $(\beta>0), \gamma$ o parâmetro de forma $(\gamma>0)$ e x o centro de classe de diâmetro $(x>0)$. A supressão do parâmetro de locação caracteriza a função hiperbólica de dois parâmetros.
Para a obtenção dos parâmetros da $f d p$ em uma idade futura, utilizou-se o sistema de equações utilizado, que se baseia no estudo proposto por Nogueira et al. (2005).

$$
\begin{gathered}
\beta_{2}=\beta_{1}\left(\frac{I_{1}}{I_{2}}\right)+\alpha\left(1-\frac{I_{1}}{I_{2}}\right) \\
\operatorname{Ln} \gamma_{2}=\operatorname{Ln} \gamma_{1} \mathrm{e}^{\left(-\left(\alpha_{0}\right)\left(I_{2}^{\alpha_{1}}-I_{1}^{\alpha_{1}}\right)\right)} \\
\mathrm{N}_{2}=\mathrm{N}_{1} \mathrm{e}^{\left(-\left(\alpha_{0}\right)\left(\mathrm{I}_{2}^{\alpha_{1}}-\mathrm{I}_{1}^{\alpha_{1}}\right)\right)}
\end{gathered}
$$

em que $I_{1}$ e $I_{2}$ são as idades atual e futura, respectivamente, em meses; $\gamma_{1}$ e $\gamma_{2}$ são os parâmetros de forma da função hiperbólica nas idades atual e futura; $\beta_{1}$ e $\beta_{2}$ são os parâmetros de escala da função hiperbólica nas idades atual e futura; $N_{1}$ e $N_{2}$ são as frequências de árvores por hectare nas idades atual e futura; $L n$ indica o logaritmo neperiano; e $a_{0}$ e $a_{1}$ são os parâmetros dos modelos.

O modelo de AC e o modelo de distribuição diamétrica foram empregados para projetar a distribuição de diâmetros para todas as idades. Gráficos da relação entre valores observados e estimados foram feitos para todas essas projeções. Para a avaliação da exatidão das projeções foi utilizado o procedimento estatístico proposto por Leite e Oliveira (2002) a 5\% de probabilidade. Avaliaram-se graficamente as projeções entre valores estimados e projetados pelo MDD e pelos modelos de AC.

\section{RESULTADOS}

Entre todas as redes treinadas, as redes do tipo $M L P$ apresentaram melhores resultados, quando comparadas com as redes do tipo RBF e Perceptron, para todas as formatações dos arquivos de dados, sendo as arquiteturas das duas melhores, em função do erro de treinamento, para cada forma de arquivo, apresentadas na Tabela 2 .

Para todas as simulações e idades, ajustou-se a função hiperbólica de dois parâmetros, e os ajustes apresentaram aderência aos dados pelo teste de aderência Kolmogorov-Smirnorv com 5\% de probabilidade. $\mathrm{O}$ teste $\mathrm{t}$ foi significativo para todos os parâmetros em todas as equações do sistema. Todas as equações apresentaram valores reativamente altos de coeficientes de correlação entre valores observados e estimados, indicando que as variáveis

Revista Árvore, Viçosa-MG, v.36, n.5, p.931-939, 2012 


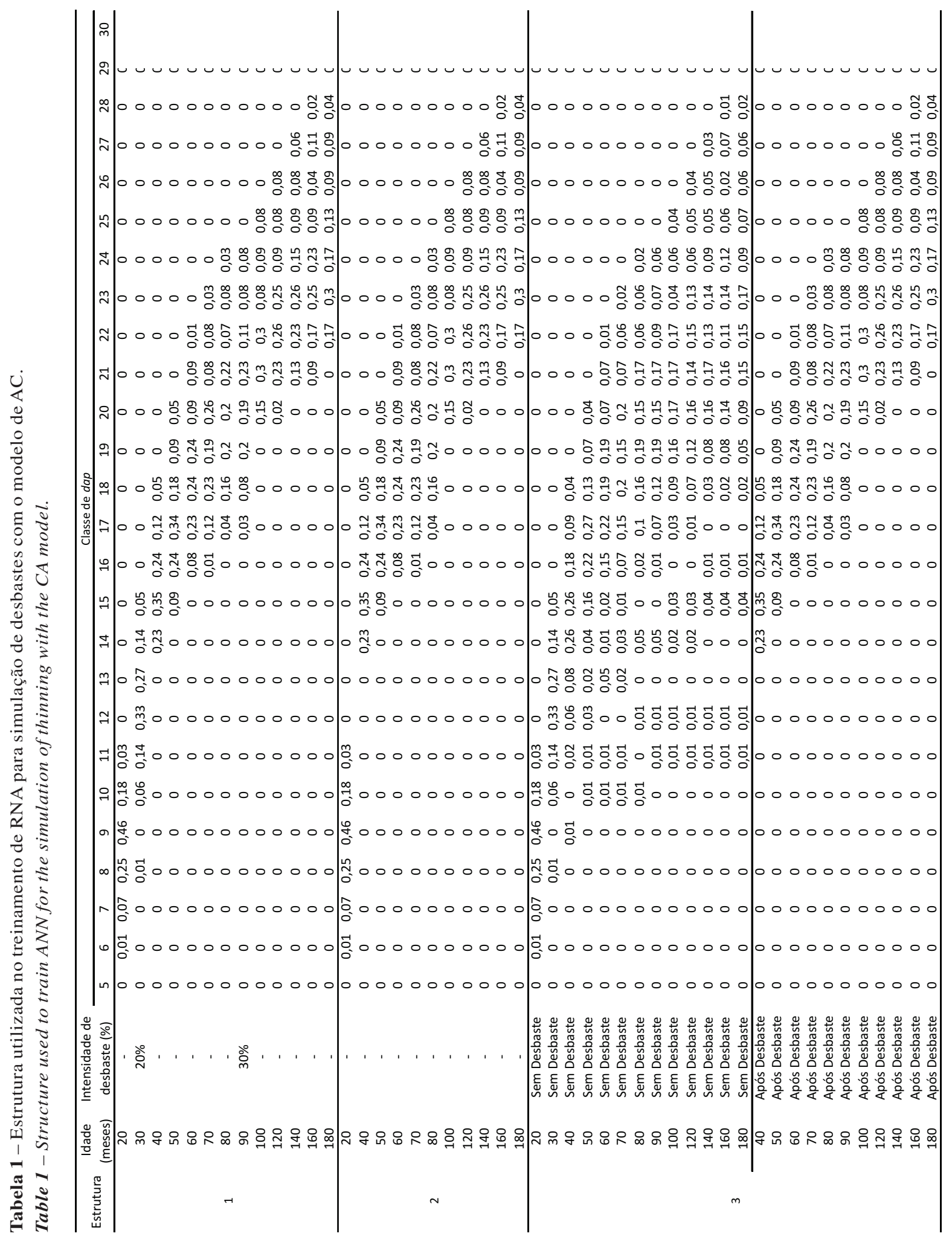

Revista Árvore, Viçosa-MG, v.36, n.5, p.931-939, 2012 
Tabela 2 - Principais características de duas redes neurais artificiais treinadas para avaliar a evolução da distribuição diamétrica de povoamentos de eucalipto em duas formas de entrada de dados.

Table 2 - Main characteristics of two artificial neural network trained to evaluate the evolution of diameter distribution of eucalyptus in two forms of data entry.

\begin{tabular}{|c|c|c|c|c|c|c|}
\hline \multirow[b]{2}{*}{ Arquivo } & \multirow[b]{2}{*}{ Rede } & \multirow{2}{*}{$\begin{array}{c}\mathrm{N}^{\mathrm{o}} \mathrm{de} \\
\text { Neurônios de } \\
\text { intermediários }\end{array}$} & \multirow{2}{*}{$\begin{array}{c}\mathrm{N}^{\circ} \text { de } \\
\text { Neurônios de } \\
\text { entrada }\end{array}$} & \multirow{2}{*}{$\begin{array}{c}\mathrm{N}^{\circ} \mathrm{de} \\
\text { Neurônios de } \\
\text { saída }\end{array}$} & \multicolumn{2}{|c|}{ Função de Ativação } \\
\hline & & & & & Camada Oculta & Camada de Saída \\
\hline \multirow{2}{*}{1} & Rede-1 & 8 & 11 & 1 & Exponencial & Seno \\
\hline & Rede-2 & 8 & 11 & 1 & Exponencial & Logistica \\
\hline \multirow{2}{*}{2} & Rede-1 & 7 & 10 & 1 & Exponencial & Logistica \\
\hline & Rede-2 & 7 & 12 & 1 & Exponencial & Seno \\
\hline \multirow{2}{*}{$3 \mathrm{~A}$} & Rede-1 & 7 & 9 & 1 & Tangente Hiperbólica & Exponencial \\
\hline & Rede-2 & 7 & 10 & 1 & Logistica & Tangente Hiperbólica \\
\hline \multirow{2}{*}{$3 \mathrm{~B}$} & Rede-1 & 7 & 11 & 1 & Exponencial & Logistica \\
\hline & Rede-2 & 7 & 12 & 1 & Exponencial & Identidade \\
\hline
\end{tabular}

independentes utilizadas nas equações têm grande influência nas variáveis dependentes. A análise gráfica dos resíduos permitiu inferir a inexistência de tendenciosidade nos ajustes (Figura 1). Os modelos ajustados e respectivos coeficientes de correlação são apresentados a seguir:

$$
\begin{aligned}
\beta_{2} & =\beta_{1}\left(\frac{I_{1}}{I_{2}}\right)+23,5565\left(1-\frac{I_{1}}{I_{2}}\right) \quad R_{\hat{y} y}=0,964 \\
\operatorname{Ln} \gamma_{2} & =\operatorname{Ln} \gamma_{1} \mathrm{e}^{\left(-(0,1687)\left(I_{2}^{0,2564}-I_{1}^{0,2564}\right)\right)} \quad \mathrm{R}_{\hat{y} y}=0,954 \\
N_{2} & =N_{1} \mathrm{e}^{\left(-(14,2704)\left(I_{2}^{-0,0104}-I_{1}^{-0,0104}\right)\right)} \quad R_{\hat{y y}}=0,995
\end{aligned}
$$

Com as duas melhores redes obtidas para cada arquivo e com o modelo de distribuição diamétrica, foram feitas as projeções das distribuições diamétricas no arquivo de parcelas destinado à validação do modelo. Resultados da aplicação do procedimento proposto por Leite e Oliveira (2002) são apresentados na Tabela 3. Esses resultados indicaram que as regras de evolução representaram a dinâmica do fenômeno estudado com eficiência e exatidão.

A avaliação gráfica entre valores observados e estimados foi realizada para as melhores redes de cada forma de ajuste. Na Figura 2 são apresentadas as análises gráficas de uma parcela escolhida aleatoriamente, com um desbaste aos 80 meses com $30 \%$ e outro com desbastes na idade de 120 meses com 50\%.

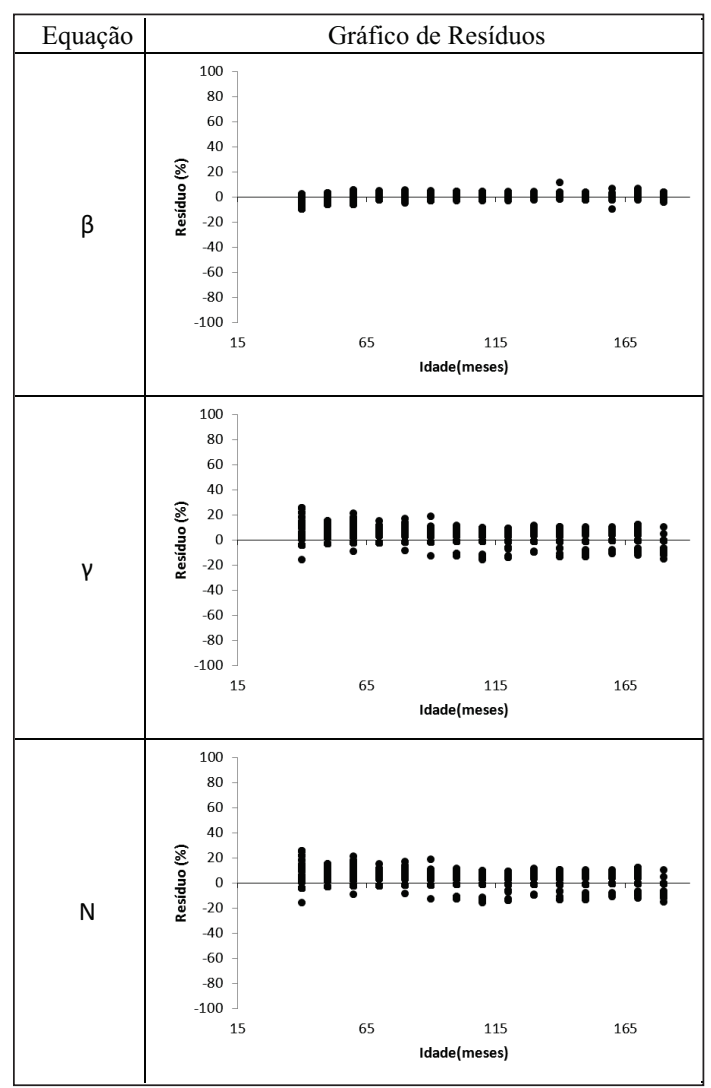

Figura 1 - Dispersão dos resíduos (\%) em função da idade nas três equações do modelo de distribuição diamétrica.

Figure 1 -Dispersion of errors (\%) according to age for three model equations diameter distribution.

Revista Árvore, Viçosa-MG, v.36, n.5, p.931-939, 2012 


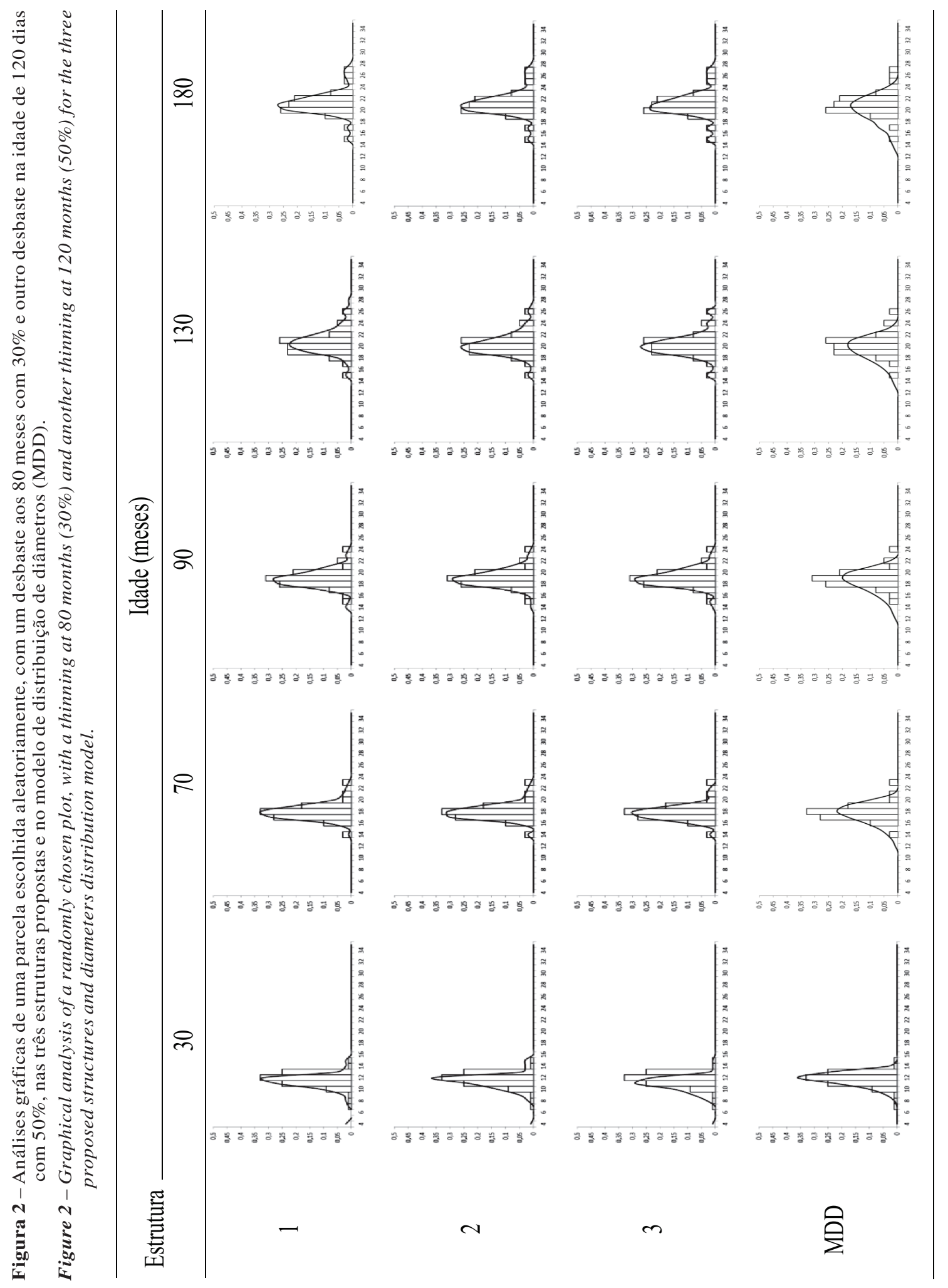

Revista Árvore, Viçosa-MG, v.36, n.5, p.931-939, 2012 
Tabela 3 - Resumo dos resultados obtidos com o procedimento estatístico proposto por Leite e Oliveira (2002) para as melhores RNA e para o modelo de distribuição diamétrica $(\alpha=5 \%)$, com o resultado da avaliação da hipótese $\mathrm{H}_{0}$ : a distribuição de diamétrica estimada é igual à projeção estimada.

Table 3 - Summary of results obtained with the statistical procedure proposed by Leite and Oliveira (2002), for the best ANN model and the diameter distribution $(\alpha=5 \%)$, with the result of evaluation of the hypothesis $H_{0}$ : The estimated diameter distribution is equal to the estimated projection.

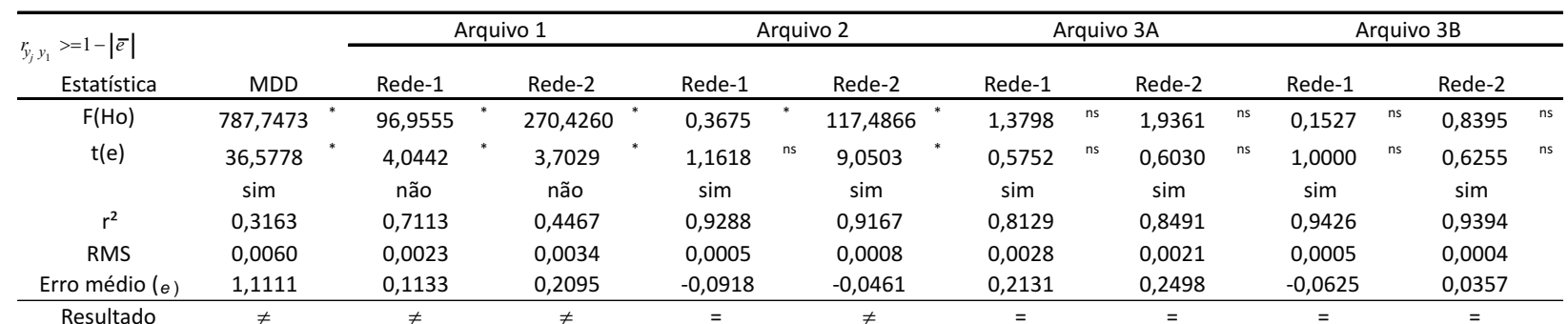

\section{DISCUSSÃO}

A modelagem de distribuições de diâmetros em povoamentos de eucalipto tem sido feita com base em predição e projeção de parâmetros de uma distribuição estatística, empregando modelos de regressão (NOGUEIRA, et al., 2005). Diferentes tipos de distribuições estatísticas já foram empregados para descrever a estrutura diamétrica de povoamentos florestais, como: Gama (NELSON. 1964), log-normal (BLISS; REINKER, 1965), Beta (CLUTTER; BENNETT, 1965), Johnson's SB (HAFLEY; SCHUREUDER, 1977; PALAHÍ et al., 2007) e a distribuição Weibull (WEIBULL, 1951; BAILEY; DELL, 1973; PALAHÍ et al., 2007). Desde 1973, a função Weibull tem sido amplamente difundida e utilizada na área florestal (CLUTTER; ALLISON, 1974; HAFLEY; SCHREUDER, 1977; MATNEY; SULLIVAN, 1982; CAO, 2004; NOGUEIRA et al., 2005; PALAHÍ et al., 2006; PALAHÍ et al., 2007), contudo os trabalhos de Guimarães (2002) e Leite et al. (2010) demonstraram a superioridade da função hiperbólica, para a descrição da distribuição diamétrica de povoamentos equiâneos.

A eficiência das estimativas geradas pelos modelos de distribuição de diâmetros usuais é dependente da qualidade dos dados utilizados e da qualidade do ajuste das equações que compõem o modelo. Cada uma das equações desses sistemas tem um erro associado, sendo a estimativa dos parâmetros da função estatística dependente da exatidão das equações que compõem o sistema.

Neste estudo foi proposto um método alternativo para modelagem de distribuições diamétricas e simulações de desbaste que não envolvam o uso de modelos de regressão para estimar a proporção de árvores por classe de diâmetro. A proposta consiste em associar um modelo de autômatos celulares e redes neurais artificiais. É uma abordagem simples que exige apenas distribuições de diâmetros observadas e se baseia simplesmente no fato de a probabilidade de cada classe de diâmetro em uma idade futura ser dependente do número atual de árvores nessa classe e nas classes vizinhas. Por esse fato, as árvores suprimidas apresentam melhor representação, como pode ser observado na Figura 2.

As três diferentes metodologias propostas diferiram na forma de ajuste e simulação de desbastes. A primeira estrutura apresenta como principal característica a projeção e simulação de desbaste completa com apenas uma estrutura, ou seja, uma única rede é responsável pela projeção e desbaste, e este deve ser definido como variável de entrada para o modelo. A presença do desbaste como variável de entrada é determinada pela presença de oito neurônios na camada de entrada (Tabela 2). Com a segunda metodologia, a simulação dos desbastes deve ser feita pelo usuário, e toda a projeção deve ser realizada por única rede. Dessa forma, uma única rede deve captar as diferentes tendências de crescimento expressas pelo povoamento antes e depois da realização do desbaste. Na terceira forma de modelagem, as diferentes tendências de crescimento, existentes antes e depois do desbaste, são expressas por diferentes redes, e esse fato apresenta considerável melhoria na descrição da dinâmica de crescimento, o que pode ser observado nas estatísticas do teste L\&O (Tabela 3). Por esse fato, recomenda-se a utilização da terceira metodologia para a projeção da distribuição diamétrica de povoamentos de eucalipto desbastados.

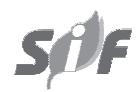

Revista Árvore, Viçosa-MG, v.36, n.5, p.931-939, 2012 
Conforme resultado deste estudo, o enfoque de modelagem resulta em estimativas livres de bias, podendo ser utilizado para a projeção da distribuição diamétrica de povoamentos submetidos a desbastes.

\section{REFERÊNCIAS}

AXTELL, R.; EPSTEIN, J. M. Growing artificial societies: Social science from the Rottorn Up. Washington: MIT Press, 1996.

BAILEY, R. L.; DELL, T. R. Quantifying diameter distributions with the Weibull function. Forest Science, v.19, n.2, p.97-104, 1973.

BAK, P.; CHEN, K.; TANG, C. A forest-fire model and some thoughts on turbulence. Physics Letters A, v.147, n.5/6, p.297-300, 1990.

BLISS, C. L.; REINKER, K. A. A log normal approach to diameter distributions in evenaged stands. Forest Science, v.10, n.3, p.350-360, 1964.

BRAGA, A. P.; LUDERMIR, T. B.; CARVALHO, A. P. L. F. Redes neurais artificiais: teoria e aplicações. Rio de Janeiro: 2000. 262p.

CAMpos, J. C. C.; LEITE, H. G. Mensuração florestal: perguntas e respostas. 3.ed. Viçosa, MG: Universidade Federal de Viçosa, 2009. 548p.

CAO, Q. V. Predicting parameters of a Weibull function for modeling diameter distribution. Forest Science, v.50, n.4, p.682-685, 2004.

CLUTTER, J. L.; Allison, B. J. A growth and yield model for Pinus radiata in New Zealand for tree and stand simulation. Royal College of Forestry, Research Notes, n.30, p.136-160, 1974.

CLUTTER, J. L.; BENNETT, F. A. Diameter distributions in old-field slash pine plantations. Georgia Forest Research Council Report, n.13, p.1-9, 1965.

CLUTTER, J. L. et al. Timber management: a quantitative approach. New York: John Willey \& Sons, 1983. 333p.

Revista Árvore, Viçosa-MG, v.36, n.5, p.931-939, 2012
d’HUMIERES, D.; LALLEMAND, P. Lattice gas cellular automata, a new experimental tool for hydrodynamics, Physical Review Letters, v.56, n.14, p.1505-1508, 1986.

DIAMANTOPOULOU, M. J. Artificial neural networks as an alternative tool in pine bark volume estimation. Computers and electronics in agriculture, v.48, n.3, p.235-244, 2005.

GERHARDT, M.; SCHUSTER, H. A cellular automaton describing the formation of spatially ordered structures in chemical systems. Physica D: Nonlinear Phenomena, v.36, n.3, p.209-221, 1989.

GÖRGENS, E. Estimação do volume de árvores utilizando redes neurais artificiais. 2006. 84f. Dissertação (Mestrado em Ciência Florestal) - Universidade Federal de Viçosa, Viçosa, MG, 2006.

GUIMARÃES, D. P. Uma função hiperbólica de distribuição probabilística de alta flexibilidade. Planaltina: Embrapa Cerrados, 2002. 40p.

HAFLEY, W. L.; SCHREUDER, H. T., Statistical distributions for fitting diameter and height data in ever-aged stands. Canadian Journal of Forest Research, v.7, n.1, p.184-487, 1977.

HAYKIN, S. Redes neurais: princípios e prática. Porto Alegre: Bookman, 2001. 900p.

ILACHINSKI, A. Cellular automata: a discrete universe. USA: World Scientific Publishing Company, 2001. 808p.

KESSLER, D. A.; LEVINE, H.; REYNOLDS W. N. Coupled-map lattice model for crystal growth. Physical Review A, v.42, n.10, p.6125-6128, 1990.

LEITE, H. G.; OLIVEIRA, F. H. T. Statistical procedure to test identity between analytical methods. Communications in Soil Science and Plant Analysis, v.33, n.7/8, p.1105-1118, 2002.

LEITE, H. G. et al. Avaliação do ajuste das funções Weibull e hiperbólica a dados de povoamentos de eucalipto submetidos a desbaste. Revista Árvore, v.34, n.2, p.305-311, 2010. 
LINDEMAYER, A.; ROZENBERG, G. Review of "Automata, languages, development". NorthHolland Publishing Company, v.9, n.1, p.16-18, 1976.

MATHEY, A. H. et al. Forest planning using coevolutionary cellular automata. Forest Ecology and Management, v.239, n.1/3, p.45-56, 2007.

MATNEY, T. G.; SULLIVAN, A. D. Variable top volume and height predictions for slash pine trees. Forest science, v.28, n.2, p.74-82, 1982.

NELSON, T. C. Diameter distribution and growth of loblolly pine. Forest Science, v.10, n.1, p.105-114, 1964.

NOGUEIRA, G. S. et al. Modelo de distribuição diamétrica para povoamentos de Eucalyptus sp. submetidos a desbaste. Revista Árvore, v.29, n.4, p.579-589, 2005.

PALAHÍ, M.; PUKKALA, T.; TRASOBARES, A. Modelling the diameter distribution of Pinus sylvesris, Pinus nigra and Pinus halepensis forest stands in Catalonia using the truncated Weibull function. Forestry, v.79, n.5, p.553-562, 2006.
PHIPPS, M. J. From local to global: the lesson of cellular automata. In: DEANGELIS, D. L.; GROSS, L. J. (Ed.) Individual-based models and approaches in ecology: concepts and models. London: Chapman and Hall, 1992.

SEGEL, L. A. Immunology viewed as the study of an autonomous decentralized system. In: DASGUPTA, D. Artificial immune systems and their applications. New York, SpringerVerlag, 1999.

SILVA, M. L. M. Estimação do diâmetro sem casca e diâmetro do cerne para árvores de Tectona grandis Linn. utilizando redes neurais artificiais. 2008. 47f. Monografia (Graduação Engenharia Florestal) - Universidade Federal de Viçosa, Viçosa, MG, 2008.

WOLFRAM, S. Cellular automata. London: Los Alarnos Science, 1983.1083p.

WEIBULL, W. A Statistical distribution function of wide applicability. Journal of Applied Mechanics, v.18, p.293-297, 1951. 
